UROLOGICAL ONCOLOGY

\title{
The prognostic significance of capsular incision into tumor during radical prostatectomy
}

Preston MA, Carrière M, Raju G, Morash C, Doucette S, Gerridzen RG, Bella AJ, Eastham JA, Scardino PT, Cagiannos I

Division of Urology, Department of Surgery, University of Ottawa, Ottawa, Ontario, Canada

Eur Urol. 2011; 59: 613-8

Background: The prognostic significance of capsular incision (CapI) into tumor during radical prostatectomy (RP) with otherwise organ-confined disease remains uncertain.

Objective: To evaluate the impact of CapI into tumor on oncologic outcome.

Design, Setting, and Participants: A retrospective review of 8110 consecutive patients with prostate cancer treated at Ottawa Hospital and at Memorial Sloan-Kettering Cancer Center, both tertiary academic centers, between 1985 and 2008.

Intervention: All patients underwent an open, laparoscopic or robotic RP.

Measurements: Patients were divided into four pathologic categories: group 1 (CapI group), positive surgical margins (PSMs) without extraprostatic extension (EPE); group 2, negative surgical margins (NSMs) without EPE; group 3, NSM with EPE; group 4, PSMs with EPE. Estimates of recurrence-free survival were generated with the Kaplan-Meier method. Recurrence was defined as a prostate-specific antigen (PSA) $>0.2 \mathrm{ng} / \mathrm{mL}$ and rising. Cox proportional hazards regression was used to estimate the hazard ratio (HR) for recurrence controlling for pretreatment PSA, RP date, RP Gleason sum, seminal vesicle invasion, and lymph node involvement. Pathologic categories were defined in the model by including the variables EPE and surgical margins (SMs) as well as their interaction.

Results and Limitations: Median follow-up was 37.3 mo. The 5-yr recurrence-free probability after RP for the CapI group was $77 \%$ (95\% confidence interval [CI], 72-83). This was not only inferior to patients with NSMs and no EPE $(\log$ rank $p<0.0001)$ but also to those with NSMs and EPE $(\log$ rank $p=0.0002)$. In multivariate analysis the interaction between EPE and SM was not significant $(\mathrm{p}=0.26)$. In the adjusted model excluding the interaction term, patients with EPE had an increased risk for recurrence (HR: 1.80; 95\% CI, 1.49-2.17; $\mathrm{p}<$ 0.0001 ) as did those with positive margins (HR: 1.81; 95\% CI, 1.51-2.15; $\mathrm{p}<0.0001$ ). This was a retrospective study.

Conclusions: CapI into tumor has a significant impact on patient outcome following RP. Patients, who otherwise would have organ-confined disease, will now have a higher probability of recurrence than those with completely resected extraprostatic disease.

\section{Editorial Comment}

The authors retrospectively analyzed 8110 consecutive patients from 2 tertiary centers treated by radical prostatectomy (open, laparoscopic or robotic) for prostate cancer for the prognostic impact of capsule incision into tumor.

Finally, 6855 patients were included into the analysis of which he vast majority of 5530 patients underwent a nerve-sparing procedure. 
$18 \%$ of these patients had positive surgical margins without extraprostatic extension (defined as capsule incision into tumor). Capsule incision had an independent negative prognostic impact in all groups analyzed.

This information is important to all surgeons considering nerve-sparing radical prostatectomy procedures.

\author{
Dr. Andreas Bohle \\ Professor of Urology \\ HELIOS Agnes Karll Hospital \\ Bad Schwartau, Germany \\ E-mail: boehle@urologie-bad-schwartau.de
}

\title{
Combination of adjuvant hormonal and radiation therapy significantly prolongs survival of patients with pT2-4 pN+ prostate cancer: results of a matched analysis \\ Briganti A, Karnes RJ, Da Pozzo LF, Cozzarini C, Capitanio U, Gallina A, Suardi N, Bianchi M, Tutolo M, Salonia A, Di Muzio N, Rigatti P, Montorsi F, Blute M \\ Department of Urology, Vita-Salute University, San Raffaele Scientific Institute, Milan, Italy \\ Eur Urol. 2011; 59: 832-40
}

Background: Previous prospective randomised trials have shown a positive impact of adjuvant radiation therapy (RT) in patients with locally advanced prostate cancer. However, none of these trials included patients with lymph node invasion (LNI).

Objective: The aim of this study was to assess the impact of combination adjuvant hormonal therapy (HT) and RT on the survival of patients with prostate cancer and histologically documented lymph node metastases (pN+). Design, Setting, and Participants: Data on 703 consecutive patients with LNI treated with radical prostatectomy, pelvic lymph node dissection, and adjuvant treatments between September 1986 and November 2002 at two large academic institutions were reviewed.

Measurements: For study purposes, patients treated with adjuvant HT plus RT and patients treated with adjuvant HT alone were matched for age at surgery, pathologic T stage and Gleason score, number of nodes removed, surgical margin status, and length of follow-up. Differences in cancer-specific survival (CSS) and overall survival (OS) were compared using the Kaplan-Meier method and life table analyses.

Results and Limitations: Following the matching process, 117 pT2-4 pN1 patients of $171(68.4 \%)$ treated with adjuvant HT plus RT (group 1) were compared with 247 pT2-4 pN1 patients of 532 (46.4\%) receiving adjuvant HT alone (group 2). After matching, the two groups of patients were comparable in terms of pre- and postoperative characteristics (all $\mathrm{p} \geq 0.07$ ). Mean follow-up was 100.8 mo (median: $95.1 \mathrm{mo}$; range: 3.5-229.3 mo). Overall, prostate CSS and OS rates at 5, 8 , and $10 \mathrm{yr}$ were $90 \%, 82 \%$, and $75 \%$, and $85 \%, 70 \%$, and $60 \%$, respectively. Patients treated with adjuvant RT plus HT had significantly higher CSS and OS rates compared with patients treated with HT alone at 5, 8, and $10 \mathrm{yr}$ after surgery $(95 \%, 91 \%$, and $86 \% \mathrm{vs} 88 \%, 78 \%$, and $70 \%$, and $90 \%, 84 \%$, and $74 \%$ vs $82 \%, 65 \%$, and 55\%, respectively; $p=0.004$ and $p<0.001$, respectively). Similarly, higher survival rates associated with the combination of HT plus RT were found when patients were stratified according to the extent of nodal invasion (namely, two or fewer vs more than two positive nodes; all $\mathrm{p}$ $\leq 0.006$ ). Lack of standardised HT and RT protocols represents the main limitations of our retrospective study. Conclusions: Adjuvant RT plus HT significantly improved CSS and OS of pT2-4 pN1 patients, regardless of the extent of nodal invasion. These results reinforce the need for a multimodal approach in the treatment of node-positive prostate cancer. 